\title{
Response to Letter to the Editor: An Evidence Based Algorithm for the Management of Marginal Ulcers following Roux-en-Y Gastric Bypass
}

\author{
William R. J. Carr • Kamal K. Mahawar • \\ Shlok Balupuri • Peter K. Small
}

Published online: 15 July 2014

(C) Springer Science+Business Media New York 2014

We would like to thank Dr Mrak for the opportunity to comment on his interesting point that $\mathrm{pH}$ meter should be included within the algorithm for the management of marginal ulcers. We agree the Siilin paper contributes to the argument that hyperacidity in the pouch is one of the risk factors for marginal ulceration and that a smaller pouch size results in less acid release and has the potential to reduce marginal ulcer rates [1]. Nevertheless, in the absence of any detectable marginal ulceration, we are unaware of any evidence supporting lifelong acid suppression, although this was not covered in the algorithm, which focused on patients with a diagnosis of marginal ulceration. It would, however, seem reasonable to offer a trail of proton pump inhibitor (PPI) treatment to symptomatic patients, which could be continued if beneficial. We remain unconvinced that measuring $\mathrm{pH}$ levels is likely to alter the management in these patients.

In patients with persistent marginal ulcers, hyperacidity, presumably due to a large pouch, could be an important factor. Revision of the pouch should hence form a part of the surgical strategy for some of these patients. Furthermore, whilst
Hedberg's paper does support the argument that hyperacidity has a role in the pathogenesis of marginal ulceration five out of six patients were diagnosed with marginal ulcers at 4 weeks or less after their roux en-y bypass [2]. Within our algorithm, all patients would be covered with PPI therapy for a year and so further investigation should be restricted to refractory ulceration and the algorithm followed to address risk factors and correct anatomical abnormalities.

Conflict of Interest None

\section{Reference}

1. Siilin H, Wanders A, Gustavsson S, et al. The proximal gastric pouch invariably contains acid-producing parietal cells in Roux-en-Y gastric bypass. Obes Surg. 2005;15(6):771-7.

2. Hedberg J, Hedenström H, Nilsson S, et al. Role of gastric acid in stomal ulcer after gastric bypass. Obes Surg. 2005;15(10):1375-8.
W. R. J. Carr · K. K. Mahawar · S. Balupuri • P. K. Small

Department and Institute: Bariatric Unit, Sunderland Royal Hospital,

Sunderland SR4 7TP, UK

W. R. J. Carr $(\bowtie)$

Surgical Registrar, Sunderland Royal Hospital,

Sunderland SR4 7TP, UK

e-mail:wrjcarr@me.com 